Series A

\author{
I. MATHEMATICA
}

413

\title{
DISTORTION THEOREMS \\ FOR QUASICONFORMAL MAPPINGS
}

BY

STEPHEN AGARD

H ELSINKI 1968

S U O M A L I N EN TIEDEAKA T EM I A

doi:10.5186/aasfm.1968.413 
Communicated 10 March 1967 by Olli Lehto and K. I. Virtanen 


\section{DISTORTION THEOREMS FOR QUASICONFORMAL MAPPINGS*}

The idea that quasiconformal mappings transform infinitesimal circles into infinitesimal ellipses with bounded eccentricity is quite familiar. It is certainly a consequence of Mori's estimate, [7], for a K-quasiconformal mapping $w=f(z)$ of a plane domain $D$ onto a plane domain $D^{\prime}$ : if $|\xi-\zeta|=|\eta-\zeta|$, if the disk $\{z:|z-\zeta| \leqq|\xi-\zeta|\}$ lies in $D$, and if the disk $\{w:|w-f(\zeta)| \leqq|f(\xi)-f(\zeta)|\}$ lies in $D^{\prime}$, then

$$
\left|\frac{f(\xi)-f(\zeta)}{f(\eta)-f(\zeta)}\right| \leqq e^{\tau K}
$$

Gehring, [4], has shown that a definition of quasiconformality can be based on these notions. An orientation preserving homeomorphism $f$ of a plane domain $D$ is $K$-quasiconformal, $1 \leqq K$, if and only if

$$
H_{f}(\zeta)=\lim _{\substack{\mid=r \\ r \rightarrow 0 \\ r \rightarrow 0}}\left|\frac{f(\xi)-f(\zeta)}{f(\eta)-f(\zeta)}\right|
$$

is bounded in $D$, and a.e. $\leqq K$.

A substantially different approach to quasiconformal mappings is through the Beltrami equation

$$
f_{\bar{z}}=\chi f_{z},
$$

satisfied weakly by each $K$-quasiconformal mapping $f$, with $\chi$ measurable, $|\chi(z)| \leq k<1$ a.e. in $D, \frac{1+k}{1-k}=K$. Conversely, [2], [5], given such $\chi$, there exists a weak solution $f$ of (1.1), which is $K$-quasiconformal and unique in the sense that if $g$ is another solution, $f \circ g^{-1}$ is conformal in $g(D)$. If we assume that $D$ is the finite plane, then the image $f(D)$ will also be the finite plane, and the allowable normalization $f(0)=0, f(1)=1$, assures that $f$ is unique. We will denote this unique, normalized solution of (1.1) by $f^{\chi}$.

\footnotetext{
* Research sponsored by National Science Foundation Grant NSF 5990 Stanford University.
} 
Various investigations have centered on determining the maximum for the critical ratio

$$
R=\left|\frac{f(\xi)-f(\zeta)}{f(\eta)-f(\zeta)}\right|,
$$

although it is clear that without some normalization the ratios will be unbounded even in the class of conformal (1-quasiconformal) mappings. For example, we may denote by $N_{1}$ the following problem: For $a \geq 1$, $K \geq 1$, in the class $Q_{1}$ of $K$-quasiconformal mappings $p$ of the unit disk onto itself, with $\varphi(0)=0$, find

$$
P_{1}=P_{1}(a, K)=\sup \left\{\left|\frac{\varphi(\xi)}{\varphi(\eta)}\right|:\left|\frac{\xi}{\eta}\right|=a\right\} .
$$

Shah and Fan, [10], solved problem $N_{1}$ by the method of parametric representation, [9], in the following implicit form: If $\eta$ is defined by

$$
\eta(x)=\frac{(1+x)}{2 \pi} \int_{\xi-\text { plane }} \int_{\frac{d \sigma(\xi)}{|\xi||1-\xi||\xi+x|},},
$$

then $y=P_{1}(a, K)$ is the solution to the equation

$$
\int_{a}^{y} \frac{d x}{x \eta(x)}=\log K
$$

A related problem was to determine the supremum of the numbers $H_{f}(\zeta)$, among all $K$-quasiconformal mappings $f$. For this purpose, Lehto, Virtanen, and Väisälä, [6], solved (for $a=1$ ) the following normalized problem, which we denote by $N_{2}$ : For $a \geq 1, K \geq 1$, in the class $Q_{2}$ of $K$-quasiconformal mappings $\varphi$ of the finite plane onto itself, with $\varphi(0)=0$, $\varphi(1)=1$, find

$$
P_{2}=P_{2}(a, K)=\sup \{|\varphi(\xi)|:|\xi|=a\} .
$$

This problem is of course equivalent to the problem of maximizing the ratios $R$ in (1.2), for $f K$-quasiconformal in the finite plane, with

$$
\left|\frac{\xi-\zeta}{\eta-\zeta}\right|=a .
$$

It comes as only a mild surprise that the solutions to problems $N_{1}$ and $N_{2}$ are the same. In this note, we calculate $P_{2}(a, K)$ in a reasonably explicit form. We then establish the equivalence of the problems from an abstract viewpoint, and finally show that $y=P_{2}(a, K)$ also solves (1.4). 
Our first task, however, will be to derive an integral representation for the hyperbolic density, which will be needed in the computations.

2.1. Hyperbolic densities and distances: For a domain $E$ consisting of the extended $z$-plane minus $n$ points $\left\{z_{1}, z_{2}, \ldots ; z_{n}\right\}, n \geq 3$, we represent the universal covering surface by the upper half plane $\{\operatorname{Im}(w)>0\}$, and let $z=J(w)$ be an analytic covering. We define the hyperbolic density $\varrho_{E}$ in $E$ by

$$
\varrho_{E}(z)=\left|\frac{d f(z)}{d z}\right| / \operatorname{Im}(f(z)),
$$

where $f$ is a local inverse for $J$. The right side of (2.1) is independent of both $J$ and the branch $f$. The hyperbolic distance $\sigma_{E}$, is then defined for points $Z^{\prime}, Z^{\prime \prime}$ in $E$, by

$$
\sigma_{E}\left(Z^{\prime}, Z^{\prime \prime}\right)=\inf \int_{\gamma} \varrho_{E}(z)|d z|,
$$

where the infimum is taken over the class of $\operatorname{arcs} \gamma$ joining $Z^{\prime}$ and $Z^{\prime \prime}$ in $E$, for which the integral has meaning.

In the special case $E_{0}:\left\{z_{1}, z_{2}, z_{3}\right\}=\{0,1, \infty\}, n=3$, a suitable covering $J$ is the familiar elliptic modular function, [8]. We calculate the hyperbolic density $\varrho_{E_{0}}$, hereafter referred to simply as $\varrho$, and the corresponding hyperbolic distance $\sigma$, for certain pairs of points.

2.2. The Integral Representation: Let $D$ be the domain obtained by deleting from the $z$-plane the real slits $\{z \leq 0\}$ and $\{z \geq 1\}$. For $z=r e^{i \ominus}$ $\in D,-\pi<\Theta<\pi$, set $\sqrt{z}=\sqrt{r} e^{i \theta_{2}^{2}}$. We consider the Jacobian elliptic function $\zeta=\operatorname{sn}(u, \sqrt{z})$, doubly periodic in $u$. In $D$, we may regard its primitive periods $4 K(z), 2 i K^{*}(z)$ as single valued analytic continuations of

$$
\begin{gathered}
K(z)=\int_{0}^{1} \frac{d t}{\left\{\left(1-t^{2}\right)\left(1-z t^{2}\right)\right\}^{1 / 2}}, 0<z<1, \\
K^{*}(z)=K(1-z) .
\end{gathered}
$$

In this section, we use the symbol * to denote replacement of the argument $z$ by the argument $1-z$, and ' to denote differentiation with respect to $z$, hence $\left(K^{*}\right)^{\prime}=-\left(K^{\prime}\right)^{*}$.

It is well known that $\zeta=\operatorname{sn}(u, \sqrt{z})$ maps the interior of the parallelogram $P$, whose vertices are $\pm K \pm i K^{*}$, conformally onto the $\zeta$-plane minus four analytic arcs, with 


$$
\left(\frac{d \zeta}{d u}\right)^{2}=\left(1-\zeta^{2}\right)\left(1-z \zeta^{2}\right)
$$

The area of the parallelogram $P$ is easily seen to be $4 \operatorname{Im}\left(i K^{*} \bar{K}\right)=$ $4 \operatorname{Re}\left(K^{*} \bar{K}\right)$. We therefore find

$$
\begin{aligned}
4 \operatorname{Re}\left(K^{*} \bar{K}\right) & =\iint_{P} d \sigma(u)=\iint_{\zeta-\text { plane }}\left|\frac{d u}{d \zeta}\right|^{2} d \sigma(\zeta) \\
& =\int_{\xi \text {-plane }} \int_{\mid} \frac{d \sigma(\zeta)}{\left|1-\zeta^{2}\right|\left|1-z \zeta^{2}\right|} \\
& =\frac{1}{2} \iint_{\xi-\text { plane }} \frac{d \sigma(\xi)}{|1-\xi||\xi||\xi-z|} .
\end{aligned}
$$

For the last step it must be remembered that in the transformation $\xi=1 / \zeta^{2}$, each point in the $\xi$-plane is covered twice.

If $z=J(w)$ is the elliptic modular function, then a local inverse in $\{\operatorname{Im}(z)>0\}$ is given by

$$
f(z)=i K^{*}(z) / K(z),
$$

[8], and hence in $\{\operatorname{Im}(z)>0\}$, we have

$$
\begin{aligned}
\varrho(z) & =\left|\frac{d}{d z}\left(\frac{i K^{*}}{K}\right)\right| / \operatorname{Im}\left(\frac{i K^{*}}{K}\right) \\
& =\frac{\left|K\left(K^{*}\right)^{\prime}-K^{*} K^{\prime}\right| /|K|^{2}}{\operatorname{Re}\left(K^{*} \bar{K}\right) /|K|^{2}} \\
& =\left|K\left(K^{\prime}\right)^{*}+K^{*} K^{\prime}\right| / \operatorname{Re}\left(K^{*} \bar{K}\right) .
\end{aligned}
$$

We contend that

$$
K\left(K^{\prime}\right)+K^{*} K^{\prime}=\pi / 4 z(1-z),
$$

and since both sides are analytic in $D$, it is sufficient to check for $0<z<1$, where we have the explicit representation (2.2). We use the classical formula [3] for $0<r<1$,

$$
\frac{d K\left(r^{2}\right)}{d r}=\frac{E\left(r^{2}\right)-\left(1-r^{2}\right) K\left(r^{2}\right)}{r\left(1-r^{2}\right)}
$$

where

$$
E(z)=\int_{0}^{1} \frac{\left(1-z t^{2}\right)^{1 / 2} d t}{\left(1-t^{2}\right)^{1 / 2}}
$$


Setting $z=r^{2}$, we find

$$
K^{\prime}=\frac{d K\left(r^{2}\right)}{d r} \frac{d r}{d z}=\frac{E(z)-(1-z) K(z)}{2 z(1-z)}
$$

hence

$$
\begin{aligned}
K\left(K^{\prime}\right)^{*}+K^{\prime} K^{*} & =\frac{K\left(E^{*}-z K^{*}\right)+K^{*}(E-(1-z) K)}{2 z(1-z)} \\
& =\frac{K E^{*}+K^{*} E-K K^{*}}{2 z(1-z)} .
\end{aligned}
$$

Applying Legendre's formula, $K E^{*}+K^{*} E-K K^{*}=\pi / 2$, (2.5) follows at once.

We thus obtain, from (2.3), (2.4), and (2.5), for $\operatorname{Im}(z)>0$

$$
\varrho(z)=\left\{\frac{|z||1-z|}{2 \pi} \int_{\xi-\text { plane }} \int_{|\xi| 1-\xi|| \xi-z \mid}\right\}^{-1} .
$$

Since both sides of (2.6) are unchanged if $z$ is replaced by $1-z$, the formula holds for $\operatorname{Im}(z) \neq 0$, and by continuity for $z \neq 0,1, \infty$.

2.3. An inequality: For $0<r<1$, the real ratio $\mu$, defined by

$$
\mu(r)=\frac{-i \pi f\left(r^{2}\right)}{2}=\frac{\pi K^{*}\left(r^{2}\right)}{2 K\left(r^{2}\right)},
$$

is equal to the modulus of the ring domain obtained by deleting from the unit disk the real interval $[0, r] . \mu$ is strictly decreasing, with limits $\infty, 0$ at 0,1 respectively, and its inverse will be denoted by $\mu^{-1}$.

The important inequality ([6], page 6),

$$
\varrho(z) \geq \varrho(-|z|)
$$

may be derived from (2.6) as follows: we first observe that for any circle $C$, and any complex number $w$, the integral

$$
\int_{c} \frac{|d \zeta|}{|\zeta-w|}
$$

depends only on the distance from $w$ to $C$, increasing as $w \rightarrow C$ from inside, and increasing as $w \rightarrow C$ from outside. If we denote by $C_{R}$ the circle

$$
\zeta=\frac{1}{1-R e^{i \theta}} ; 0 \leq \Theta \leq 2 \pi
$$


A. I. 413

and if $w$ is on $C_{r}$, it then follows that

$$
\int_{c_{R}} \frac{|d \zeta|}{|\zeta-w|} \leq \int_{c_{R}} \frac{|d \zeta|}{\left|\zeta-\frac{1}{1+r}\right|} .
$$

But introducing polar coordinates $\xi=R e^{i \Theta}$ in (2.6), and setting $z=r e^{i \varphi}$, we find

$$
\begin{aligned}
\frac{1}{\varrho(z)} & =\frac{|z|}{2 \pi} \int_{0}^{\infty} \frac{d R}{R} \int_{0}^{2 \pi}\left|\frac{(1-\xi)(1-z)}{(\xi-z)}\right| \frac{R d \Theta}{|1-\xi|^{2}} \\
& =\frac{|z|}{2 \pi} \int_{0}^{\infty} \frac{d R}{R} \int_{0}^{2 \pi} \frac{1}{\left|\frac{1}{1-R e^{i \theta}}-\frac{1}{1-z}\right|} \frac{R d \Theta}{\left|1-R e^{i \Theta}\right|^{2}} \\
& =\frac{r}{2 \pi} \int_{0}^{\infty} \frac{d R}{R} \int_{C_{R}} \mid \frac{|d \zeta|}{\zeta-\frac{1}{1-r e^{i \varphi}} \mid} \\
& \leq \frac{|-r|}{2} \int_{0}^{\infty} \frac{d R}{R} \int_{C_{R}}^{\left|\zeta-\frac{1}{\mid-\frac{1}{1+r}}\right|}=\frac{1}{\varrho(-r)} .
\end{aligned}
$$

We draw two conclusions: first, that the negative real axis is a geodesic for $\sigma$, and second that if $a_{i}=\left|z_{i}\right|$,

$$
\sigma\left(z_{1}, z_{2}\right) \geq \sigma\left(-a_{1},-a_{2}\right)
$$

Hence to obtain a formula for the right hand side of (2.7), we may integrate $\varrho$ along $\alpha$, the negative real axis between $-a_{1}$ and $-a_{2}$. On $\alpha,-i f^{\prime}(z)>0$ and $\operatorname{Re}(f(z))=1$, hence $\left|f^{\prime}(z)\right|=-i f^{\prime}(z)$, and $\operatorname{Im}(f(z))=-i(f(z)-1)$. We find

$$
\begin{aligned}
& \int_{\alpha} \varrho(z)|d z|=\int_{\alpha} \frac{\left|f^{\prime}(z)\right||d z|}{\operatorname{Im}(f(z))} \\
= & \mid \int_{a_{1}}^{a_{2}} \frac{f^{\prime}(-t) d t}{1-f(-t)} \\
= & |\log (1-f(-t))|_{a_{1}}^{a_{2}} \mid \\
= & \left|\log \frac{1-f\left(-a_{2}\right)}{1-f\left(-a_{1}\right)}\right| .
\end{aligned}
$$


Since the mapping $f$ satisfies the identity

$$
\frac{1}{1-f(z)}=f\left(\frac{1}{1-z}\right)
$$

we conclude

$$
\sigma\left(-a_{1},-a_{2}\right)=\left|\log \frac{\mu\left(\left\{1+a_{2}\right\}^{-1 / 2}\right)}{\mu\left(\left\{1+a_{1}\right\}^{-1 / 2}\right)}\right|
$$

2.4. Teichmüller's Theorem: A fundamental theorem of Teichmüller, [11], [1], asserts: Given $z_{0}, w_{0} \in E_{0}, K \geq 1$, there exists $\varphi \in Q_{2}$ with $\varphi\left(z_{0}\right)=w_{0}$ if and only if $\sigma\left(z_{0}, w_{0}\right) \leq \log K$. As a second application of (2.6), we use the results of Ahlfors and Bers [2] to prove the »only if» part of this theorem. We suppose that $\varphi=f^{\varkappa}$, and there is no loss in generality in assuming that $\chi$ is continuous with compact support. For $0 \leq t \leq 1$ let $f(z, t)=f^{t \chi}(z)$. Then lemmas 19 and 21 of [2] apply, and we may assert that $f(z, t)$ is differentiable in $t$, and

$$
\frac{\partial f(z, t)}{\partial t}=\left(P b_{t}\right)(w)-w\left(P b_{t}\right)(1) ; w=f(z, t),
$$

where

$$
b_{t}(w)=\frac{\chi(z)}{1-t^{2}|\chi(z)|^{2}} \frac{f_{z}(z, t)}{\bar{f}_{\bar{z}}(z, t)} ; w=f(z, t),
$$

and $P$ is the Hilbert transform,

$$
(P g)(w)=\frac{1}{\pi} \int_{\xi-\text { plane }} \int_{\text {e }} g(\xi)\left(\frac{1}{\xi}-\frac{1}{\xi-w}\right) d \sigma(\xi) .
$$

Since $\bar{f}_{\bar{z}}=\bar{f}_{z}$, we obtain from $(2.10)$ the simple inequality

$$
\sup _{w}\left|b_{t}(w)\right| \leq \frac{k}{1-t^{2} k^{2}},
$$

while (2.11) yields easily, with (2.6), the inequality

$$
\begin{aligned}
|(P g)(w)-w(P g)(1)| & =\mid \frac{w(w-1)}{\pi} \int_{\xi-\text { plane }} \int_{\xi(\xi-w)(\xi-1)} \frac{g(\xi) d \sigma(\xi)}{\xi(\xi-w)} \\
& \leq \frac{2 \sup |g|}{\varrho(w)}
\end{aligned}
$$


As a competing path from $z=f(z, 0)$ to $\varphi(z)=f(z, 1)$, we take the trace of $f(z, t): 0 \leq t \leq 1$. Evidently, using (2.9), (2.13), and (2.12),

$$
\begin{aligned}
\sigma(z, \varphi(z)) & \leq \int_{0}^{1} \varrho(f(z, t))\left|\frac{\partial f(z, t)}{\partial t}\right| d t \\
& \left.\leq \int_{0}^{1} \frac{2 k d t}{1-t^{2} k^{2}}=\log \frac{1+t k}{1-t k}\right]_{0}^{1}=\log \frac{1+k}{1-k}=\log K
\end{aligned}
$$

3.1. Problem $N_{2}$ : We now can assert that

$$
P_{2}(a, K)=\left[\mu^{-1}\left(K \mu\left(\{1+a\}^{-1 / 2}\right)\right)\right]^{-2}-1,
$$

or equivalently, in view of (2.8),

$$
\sigma\left(-a,-P_{2}\right)=\log K
$$

For let $P_{2}^{*}$ be defined by the right hand side of (3.1), which is to say

$$
\sigma\left(-a,-P_{2}^{*}\right)=\log K, P_{2}^{*} \geq a .
$$

By the »if» part of Teichmüller's theorem, there exists $\varphi^{*} \in Q_{2}$, with

$$
\varphi^{*}(-a)=-P_{2}^{*} .
$$

Consequently,

$$
P_{2} \geq\left|\varphi^{*}(-a)\right|=\left|-P_{2}^{*}\right|=P_{2}^{*} .
$$

On the other hand, given $\varphi \in Q_{2},|\xi|=a$, we find from (2.7) and Teichmüller's theorem, $\sigma(-|\varphi(\xi)|,-a) \leq \sigma(\varphi(\xi), \xi) \leq \log K=\sigma\left(-P_{2}^{*},-a\right)$. It follows that $|\varphi(\xi)| \leq P_{2}^{*}$, hence $P_{2} \leq P_{2}^{*}$, and with (3.2), the formula is verified.

3.2. Problem $N_{1}$ : For the mapping $\varphi^{*}$ of Section 3.1, and large integers $n$, let $\varphi_{n}$ be defined for $|z| \leq 1$ by

$$
\varphi_{n}(z)=f_{n}\left(\varphi^{*}(n z)\right) / a_{n},
$$

where $f_{n}$ is a conformal mapping of $\left\{\varphi^{*}(\zeta):|\zeta| \leq n\right\}$ onto $\left\{f_{n} \mid \leq a_{n}\right\}$, normalized by $f_{n}(0)=0, f_{n}(1)=1$. By virtue of this normalization, the $\left\{f_{n}\right\}$ are a normal family in $E_{0}$, and any limit function is necessarily the identity. By construction, $\varphi_{n} \in Q_{1}$, and therefore

$$
P_{1} \geq\left|\frac{\varphi_{n}(-a / n)}{\varphi_{n}(1 / n)}\right|=\left|\frac{f_{n}\left(\varphi^{*}(-a)\right)}{f_{n}\left(\varphi^{*}(1)\right)}\right|=\left|f_{n}\left(-P_{2}\right)\right| \text {. }
$$


Letting $n \rightarrow \infty$, we conclude

$$
P_{1} \geq \lim \left|f_{n}\left(-P_{2}\right)\right|=\left|-P_{2}\right|=P_{2} .
$$

On the other hand, any $\varphi \in Q_{1}$ can be extended by reflection and rotation to a mapping $\varphi_{0} \in Q_{2}$, and with corresponding ratios equal. It follows that $P_{1} \leq P_{2}$, and with (3.3), the equivalence of problems $N_{1}$ and $N_{2}$ is established.

3.3. Remark:Returning to (1.4), we see from (2.6) and (1.3) that

$$
1 / x \eta(x)=\varrho(-x)
$$

and hence, as expected,

$$
\begin{aligned}
\int_{a}^{P_{2}} \frac{d x}{x r_{l}(x)} & =\int_{a}^{P_{2}} \varrho(-x) d x=\int_{-P_{2}}^{-a} \varrho(t) d t \\
& =\sigma\left(-a,-P_{2}\right)=\log K .
\end{aligned}
$$

\section{Stanford University}

California, USA 


\section{Bibliography}

[1] Ahlfors, L. V., The modular function and geometric properties of quasiconformal mappings, Proceedings of Minnesota Conference on Complex Analysis, Springer, (1965).

[2] - - and Bers, L., Riemann's Mapping Theorem for variable metrics, Ann. Math. Vol. 72, No. 2, (1960) 385-404.

[3] Byrd, P. F. and Friedman, M. D., Handbook of Elliptic Integrals for Engineers and Physicists, Springer, Berlin, 1954.

[4] Gehring, F. W., The definitions and exceptional sets for quasiconformal mappings, Ann. Acad. Sci. Fenn. A. I. 281, (1960), 1-28.

[5] Lehto, O. and Virtanen, K. I., On the existence of quasiconformal mappings with prescribed complex dilatation, Ann. Acad. Sci. Fenn. A. I., 274 (1960), 1-24.

[6] -»- -》- and VäISÄLÄ, J., Contributions to the distortion theory of quasiconformal mappings, Ann. Acad. Sci. Fenn. A. I. 273, (1959), 1-14.

[7] MorI, A., On quasi-conformality and pseudo-analyticity, Trans. Amer. Math. Soc. 84, (1957), 56-77.

[8] Nehari, Z., Conformal Mapping, McGraw-Hill, New York, 1952.

[9] Shah Dao-Shing, Parametric representation of quasiconformal mappings (Russian), Science Record, New Ser. III, (9), (1959), 400-407.

[10] - - and FAN LE-LE, On the modulus of quasiconformal mappings, Science Record, New Ser. 4, (5), (1960), 323-328.

[11] Teichmüller, O., Extremale quasikonforme Abbildungen und quadratische Differentiale, Abh. Preuss. Akad. Wiss. 22, (1940), 1-197. 